\title{
RADIOLOGIA E ATUAÇÃo DO TECNÓlOGO NA MEDICINA VETERINÁRIA
}

\author{
RADIOLOGY AND PERFORMANCE OF THECHNOLOGIST IN \\ VETERINARY MEDICINE
}

\author{
Danielle Muniz Pessoa Aires \\ Mestre, Docente do Curso Superior de Tecnologia em Radiologia, Faculdade \\ Evangélica de Ceres, Ceres-GO. daniellempessoa@ hotmail.com
}

\section{Endereço para correspondência:}

Rua 30, quadra 53, lote 7C, Centro. Rialma - GO. Brasil. CEP: 76310-000.

\section{RESUMO:}

INTRODUÇÃO: Os meios de diagnóstico por imagem são de grande utilidade na clínica médica veterinária, auxiliando na agilidade e precisão do diagnóstico. Este tipo de serviço possui como desafio a necessidade de conhecimento específico em função da variedade de espécies. OBJETIVO: Discorrer sobre a radiologia veterinária, enfatizando a atuação do tecnólogo em radiologia na área. METODOLOGIA: Realizou-se uma revisão de literatura do tipo narrativa, através de livros e artigos publicados nas bases de dados virtuais SCIELO, Google Acadêmico e PUBVET. RESULTADOS E DISCUSSÃO: Exames de radiodiagnóstico são importantes na clínica médica de animais e que vem sendo modernizados a partir do desenvolvimento de novas tecnologias. A radiografia ainda é o exame de imagem mais utilizado no setor, pelo baixo custo e fácil acesso, no entanto, outros métodos como ultrassonografia, ressonância magnética e tomografia computadorizada também são utilizados em animais. São alguns fatores limitantes da área a disponibilidade dos serviços, a inexistência de parâmetros específicos e a escassez de estudos, considerando a diversidade de espécies animais. A legislação garante ao tecnólogo ou o técnico em radiologia a possibilidade de atuação podem atuar no radiodiagnóstico, inclusive na área veterinária, sendo recomendado treinamento específico através de estágios ou cursos. CONCLUSÃO: A radiologia veterinária é importante para a clínica médica de animais, sendo a radiografia convencional o exame de imagem mais utilizado no setor. O tecnólogo em radiologia pode atuar no radiodiagnóstico veterinário.

Palavras chave: Diagnóstico por imagem. Raio X. Veterinária. 


\begin{abstract}
:
INTRODUCTION: Imaging diagnostic means are very useful in the veterinary medical clinic, helping in the agility and precision of the diagnosis. This type of service has as a challenge the need for specific knowledge due to the variety of species. OBJECTIVE: To discuss veterinary radiology, emphasizing the role of the radiologist in the field. METHODOLOGY: A narrative literature review was conducted through books and articles published in the virtual databases SCIELO, Google Scholar and PUBVET. RESULTS AND DISCUSSION: Radiodiagnostic tests are important in the animal medical clinic and have been modernized based on the development of new technologies. Radiography is still the most used imaging exam in the industry, due to its low cost and easy access. However, other methods such as ultrasound, magnetic resonance and computed tomography are also used in animals. Limiting factors of the area are the availability of services, the lack of specific parameters and the scarcity of studies, considering the diversity of animal species. The legislation guarantees to the technologist or technician in radiology the possibility of acting can act in the radiodiagnostic, including in the veterinary area, being recommended specific training through internships or courses. CONCLUSION: Veterinary radiology is important for the medical animal clinic, and radiography is the most used imaging exam in the sector. The technologist in radiology can work in veterinary radiology.
\end{abstract}

Key words: Diagnosis by image. Technologist. X-ray.

\title{
1. INTRODUÇÃO
}

A radiologia é definida como a área da ciência que estuda as estruturas internas por meio de imagens formadas por feixes de radiação ionizante, eletromagnética ou sonora (KEALY et al., 2012). Na medicina veterinária, a radiologia é fundamental no diagnóstico das mais diversas patologias dos animais, sendo útil ainda, na realização de procedimentos cirúrgicos e na área de pesquisa (THRALL, 2014).

Entre os diversos métodos de diagnóstico por imagem utilizados na radiologia veterinária destaca-se a radiografia convencional, por se trata de um método que permite obtenção de imagens de qualidade e de baixo custo. É, portanto, o mais acessível a clínicas do setor e proprietários de animais (ANDRADE, 2007).

O aumento da demanda na área da radiologia veterinária vem exigindo do mercado mudanças para atender as peculiaridades da área. Tal fato possibilitou o desenvolvimento de equipamentos especializados, como a utilização de aparelhos móveis e portáteis que atendem a necessidade de realização de exames a campo em animais de grande porte (BARROSO; DE PAULA e ÁVILA, 2005). 
Para atender essa demanda é necessário ainda, um perfil profissional com conhecimentos específicos da área, para atuação junto ao atendimento das várias espécies animais. Conforme a Resolução do Conselho Nacional de Técnicos em Radiologia $n^{\circ} 02$ de 10 de maio de 2005 é atribuição do técnico ou tecnólogo em radiologia a realização do exame radiográfico, inclusive em medicina veterinária.

O objetivo deste estudo é discorrer sobre a radiologia veterinária, enfatizando a atuação do tecnólogo em radiologia na área.

\section{METODOLOGIA}

O presente estudo foi elaborado a partir de uma revisão de literatura narrativa realizada em julho de 2019, a partir de livros, legislação nacional, trabalhos publicados em repositórios e, principalmente, artigos científicos relevantes para a temática da pesquisa, publicados nas bases de dados virtuais Google Acadêmico, SCIELO e Pubvet. As palavras utilizadas na busca foram "diagnóstico por imagem", "radiologia veterinária", "tecnólogo" e "técnico". Foram priorizados os trabalho publicados em língua portuguesa e inglesa. Dos 23 artigos encontrados, foram selecionados 14 para a confecção do trabalho, sendo considerados como critérios de inclusão a abordagem voltada à atuação profissional e ao radiodiagnóstico veterinário.

\section{A RADIOLOGIA NA ÁREA DA MEDICINA VETERINÁRIA}

A radiologia é a especialidade médica que usa as formas de radiação (ionizante, sonora ou magnética), no processo de formação de imagens (MARCHIORI e SANTOS, 2015). O estudo radiológico é indispensável à rotina clínica da medicina veterinária, devido a sua simplicidade e rapidez na elucidação dos casos clínicos, permitindo avaliação de estruturas internas o que auxilia o diagnóstico das mais diversas patologias, como as recorrentes fraturas (OKUNO e YOSHIMURA, 2010).

A medicina veterinária vem passando por evoluções na área de diagnóstico por imagem, e é mais frequentemente utilizada no atendimento clínico de pequenos animais, como auxiliar em procedimentos cirúrgicos ou mesmo na área de pesquisa. Além do atendimento a animais domésticos, a solicitação de exames imaginológicos para animais selvagens pelos clínicos da área vem aumentando significativamente (BORTOLINI et al., 2013). 
O aprimoramento da radiologia veterinária nos últimos anos tem sido impulsionado pelas melhorias na tecnologia e o consequente desenvolvimento de novos equipamentos, como na radiografia computadorizada, principalmente pela disponibilização de sistemas menores, mais acessíveis quanto à mobilidade. Radiologistas especializados em atendimento a equinos, por exemplo, têm sido precursores no uso da tecnologia de radiografia computadorizada, devido vantagens em mobilidade para atendimentos a campo (MATHOON e SMITH, 2004).

No entanto, a área esbarra em fatores limitantes, como a disponibilidade do serviço ser comumente nas grandes cidades e ainda, a inexistência ou a pouca quantidade de estudos e parâmetros específicos para a área. Bortolini et al. (2013), aponta como entrave ao uso das técnicas em animais selvagens, a escassez de estudos específicos, considerando a grande variação anatômica entre esses animais.

A radiografia apresenta-se como o meio de radiodiagnóstico por imagem mais acessível e de baixo custo na clínica médica veterinária (PINTO et al., 2010). É destaque entre os demais métodos, por ser um exame não invasivo, indolor e que proporciona a visualização de estruturas internas, como os órgãos. Permite, portanto, a identificação de alterações comuns, como inflamações, fraturas e luxações (BARROSO; DE PAULA; ÁVILA, 2005).

O sistema osteoarticular e sistema cardiopulmonar são os mais solicitados em exames radiográficos pelos médicos veterinários. A radiografia de tórax, por exemplo, pode fornecer informações diagnósticas relevantes das doenças pulmonares, também no diagnóstico das doenças do mediastino e do tórax ósseo, sendo um dos procedimento mais frequentemente realizado em muitos departamentos (RAMPAZZO et al., 2013).

A utilização do exame radiográfico também é essencial na odontologia veterinária, área que tem crescido muito nos campos acadêmico, tecnológico, científico e médico. Aproveitando o aporte tecnológico da radiologia humana, a odontologia veterinária conta com uso da radiografia digital, dispondo de equipamento e material radiográfico especializado de última geração (MARTINEZ et al, 2009).

A ultrassonografia,é outro método de diagnóstico por imagem também já utilizado no atendimento veterinário, principalmente na área reprodutiva, como nos procedimentos de diagnóstico de gestação. Trata-se também de um exame simples, de realização rápida, seguro, não invasivo, de baixo custo e que permite obtenção de imagens em tempo real (FELICIANO et al., 2008; CARVALHO, CHAMMAS e CERRI, 2008). 
Técnicas como a ressonância magnética, tomografia computadorizada e cintilografia nuclear são aplicadas com menos regularidade, devido ao seu custo ser alto e haver pouca disponibilidade dos aparelhos na área da medicina veterinária (BORTOLINI et al., 2013).

Em animais, a tomografia computadorizada é o exame de escolha para avaliação de afecções encefálicas, por permitir a visualização do conteúdo craniano, havendo um crescimento da utilização desse exame nessa área (LORIGADOS e PINTO, 2013). Pode ser exame útil na varredura de regiões tumorais, como quando se faz planejamento de tratamento radioterápico de tumores (BIRCHARD e SHERDING, 2008).

A ressonância magnética possibilita a obtenção de imagens em planos diversificados, com a possibilidade para diferenciar detalhes sutis nos tecidos moles. Entretanto, é uma técnica de elevado custo, dependente de profissional altamente especializado, conhecedor dos cortes seccionais e de anatomia animal, o que ainda limita o uso da técnica na área animal (HAGE et al., 2010).

A radioterapia, método que faz uso da radiação no tratamento de câncer também já é empregada na medicina veterinária. Encontra-se em literatura registros do uso deste tipo de tratamento na área do ano de 1906. Animais submetidos à radioterapia têm demonstrando boa tolerância ao tratamento, com melhoria na qualidade de vida, aumento na sobrevida, e redução nos custos em quimioterápicos. No Brasil, apesar da radioterapia veterinária ainda não ser uma opção efetivamente praticada, as pesquisas envolvendo essa modalidade terapêutica em animais estão crescendo (FERNANDES et al., 2010, VETTORATO et al., 2017).

\section{ATUAÇÃO DO TECNÓLOGO NA RADIOLOGIA VETERINÁRIA}

Assim como na prática em radiologia humana, é necessária a atuação de profissionais especializados durante a rotina dos procedimentos de diagnóstico por imagem na medicina veterinária. Segundo o artigo $3^{\circ}$ da Resolução CONTER N. ${ }^{\circ}$ 02, de 10 de maio de 2005, que institui e normatiza as atribuições dos Profissionais Técnicos e Tecnólogos em Radiologia, o radiodiagnóstico veterinário é área de atuação do tecnólogo ou técnico em radiologia.

As faculdades e universidades de medicina veterinária são os principais empregadores dos profissionais da radiologia que atuam nessa área, nos hospitais escola. Em geral, tais profissionais são treinados por médicos veterinários, passaram por 
estágios específicos em radiodiagnóstico veterinário ou realizaram curso profissionalizante no setor. No entanto, não existem dados sobre a contratação em escala desses profissionais (TEODORO, 2007; ANDRADE, 2007).

A atuação de profissional da radiologia na área veterinária depende de habilidades e conhecimento específicos, já que se trabalha com uma diversidade grande de espécies. Um aspecto importante, por exemplo, é o conhecimento de métodos de contenção, em função dos pacientes não serem colaborativos e necessitarem de acompanhamento. A contenção do animal intenciona mantê-lo parado na posição adequada ao procedimento, evitando alterações, repetições ou mesmo acidentes. Pode ser realizada a contenção física, através do uso de força física pessoal e de equipamentos como mordaças, focinheiras ou laços, e ainda, a contenção química, a qual se utiliza de substâncias (sedativos e/ou anestésicos) administradas por via oral, venosa e intramuscular (OLIVA, 2014).

Aspectos fundamentais de radioproteção também devem ser observados nos procedimentos de radiodiagnóstico veterinário. Considera-se que o risco dos profissionais envolvidos na realização desses exames é pequeno, mas não pode ser subestimado. Os níveis de radiação a que esses trabalhadores são expostos devem ser tão baixos quanto possível para a realização dos procedimentos e obtenção do diagnóstico pretendido (GINJA E FERREIRA, 2002). Melo (2009) ressalta que a otimização de técnicas empregadas atualmente nos serviços radiológicos, como a radiologia computadorizada, possibilitou uma redução de doses em pacientes e acompanhantes.

Vale destacar que não se encontra na literatura valores de referência de doses para as espécies e são escassos trabalhos científicos neste sentido. Além disso, não há no Brasil norma específica à radioproteção em serviços de radiodiagnóstico veterinário, sendo utilizada como base a Portaria No 453 da Secretária de Vigilância Sanitária, do Ministério da Saúde, mesma norma base para humanos (BRASIL, 1998). Tais aspectos tornam ainda maior o desafio da rotina clínica do serviço de radiologia veterinária.

\section{CONSIDERAÇÕES FINAIS}

A radiologia é de grande relevância para a clínica médica veterinária, na identificação das patologias dos animais, configurando uma área promissora e que vem 
se modernizando, principalmente com a evolução de novas tecnologias. A radiografia ainda é o exame de imagem mais utilizado no setor, principalmente em função do fácil acesso e baixo custo, no entanto, outras técnicas, como tomografia computadorizada e até mesmo o tratamento radioterápico, já são uma realidade.

Para garantia da obtenção de exame de qualidade, realizado de maneira correta, considerando as diversas espécies de animais, é importante que o profissional seja capacitado para tal. De acordo com a legislação, o tecnólogo ou o técnico em radiologia podem atuar no radiodiagnóstico veterinário, sendo fundamental que estes profissionais possuam conhecimentos específicos para atuação na área.

\section{REFERÊNCIAS}

ANDRADE, S. A. F. Atuação do tecnólogo em radiologia na área da medicina veterinária. Revista UNILUS Ensino e Pesquisa, v. 4, n. 7, julho/dezembro.2007.

BARROSO, V.; DE PAULA, T. M.; ÁVILA JR, R . Radiologia torácica. Revista eletrônica de Veterinária REDVET. v. VI, n. 3, mar. 2005.

BIRCHARD, S. J.; SHERDING, R. G. Manual Saunders Clínica de Pequenos Animais. $2^{a}$ Ed. São paulo, Editora Roca, 2003. 2072 p.

BORTOLINI, Z.P.M.; MATAYOSHI, R.V.; SANTOS, D.P.; DOICHE, V.M.V.; MACHADO, C.R.; TEIXEIRA, L.C. Casuística dos exames de diagnóstico por imagem na medicina de animais selvagens 2009 a 2010. Arquivo Brasileiro de Medicina Veterinária e Zootecnia, v.65, n.4, p.1247-1252, 2013. Disponível em: < http://www.scielo.br/pdf/abmvz/v65n4/42.pdf> Acesso em: 22 jul. 2019.

BRASIL. Portaria n. ${ }^{\circ} 453$, de 01 de junho de 1998. Aprova as Diretrizes Básicas de Proteção Radiológica. Diário Oficial da União, 02 jun. 1998.

CARVALHO, C.F.; CHAMMAS, M.C.; CERRI, G.G. Princípios físicos do Doppler em ultrassonografia. Ciência Rural, v.38, n.3, p. 872-879, 2008. Disponível em: $<$ http://www.scielo.br/scielo.php?pid=S010384782008000300047\&script=sci_abstract $\&$ tlng=pt> Acesso em: 22 jul. 2019. 
CONTER. CONSELHO NACIONAL DE TÉCNICOS EM RADIOLOGIA. Resolução CONTER No 2, 10 de Maio de 2005. Disponível em:

<http://conter.gov.br/uploads/legislativo/n._022005.pdf > Acesso em: 22 jul.2019.

FELICIANO, M.A.R.; VICENTE, W.R.R.; LEITE, C.A.L.; SILVEIRA, T. Abordagem ultrassonográfica da neoplasia mamária em cadelas: Revisão de literatura. Revista Brasileira de Reprodução Animal, v.32, n.3, p.197-201, jul/set. 2008. Disponivel em: $<$ http://www.cbra.org.br/pages/publicacoes/rbra/download/RB163\%20Feliciano\%20vr2 \%20pag197-201.pdf> Acesso em: 23 jul. 2019.

FERNANDES, M. A. R.; ANDRADE, A. L.; LUVIZOTO, M. C. R.; Pierô, J. R.; CIARLINI, L. D. R. P. Radioterapia em Medicina Veterinária - Princípios e Perspectivas. Revista Brasileira de Física Médica, v.4, n.2, p.11-4, 2010. Disponível em:

<http://www.rbfm.org.br/rbfm/article/view/69/v4n2p11> Acesso em: 22 jul. 2019. GINJA, M.M.D.; FERREIRA, A.J.A. Efeitos biológicos da radiação X e radioproteção em medicina veterinária. Revista Portuguesa de Ciências Veterinárias, v.97, p.101109, 2002. Disponível em: < http://www.spcv.pt/download/Vol97/Vol97-n543.pdf.> Acesso em: 18 jul. 2019.

HAGE, M. C. F.N.S.; IWASAKI, M.; RABBANI, S.R.; KAMIKAWA, L.; CERVANTES, H.J.R.; BOMBONATO, P.P.; STERMAN, F.A.; OTADUY, M.C.G. Imagem por ressonância magnética na investigação da cabeça de cães. Pesquisa veterinária brasileira, v.30, n.7, p.593-604, 2010. Disponível em: <http://www.scielo.br/pdf/pvb/v30n7/a14v30n7.pdf> Acesso em: 18 jul. 2019.

KEALY, J. K.; MCALLISTER, H.; GRAHAM, J. P. Radiologia e Ultrassonografia do Cão e do Gato. São Paulo: Elsevier, 2012.

LORIGADOS, C.A.B.; PINTO, A.C.B.F. Tomografia computadorizada do encéfalo do cão: aspectos da normalidade e correlação anatômica. Arquivo Brasileiro de Medicina Veterinária e Zootecnia, v.65, n.3, p.729-734, 2013. Disponível em:

<http://www.scielo.br/pdf/abmvz/v65n3/18.pdf> Acesso em: 18 jul. 2019.

MARCHIORI, E.; SANTOS, S. L. Introdução à Radiologia. Rio de Janeiro: Guanabara Koogan, 2015.

MARTINEZ, L. A. V.; FRAZÃO, P. J. R.; FERREIRA, E. T. T.; COSTA, C.; GIOSO, M. A. Descrição das técnicas radiográficas intraorais utilizadas na medicina veterinária. Revista do Instituto de Ciências da Saúde, v. 27, n. 1, p. 39-43, 2009. 
MATTOON, J.S; SMITH, C. Breakthroughs in Radiography: Computed Rad i ography. Compendium on continuing education for the practising veterinarian, v. 16, p-58-66, 2004.

MELO, C. S. Estudo de doses e otimização de carta técnica na transição de radiologia convencional para computacional em diagnósticos veterinários. 2009. 51f. Trabalho de Conclusão de Curso (Graduação em Física Médica) - Instituto de Biociências, Universidade Estadual Paul ista, Botucatu.

OLIVA, V. N. L. S. Contenção química: contenção química de cães e gatos. In: Feitosa, Francisco Leydson. Semiologia Veterinária: A arte do Diagnóstico. São Paulo: Roca , 2014.

OKUNO, E.; YOSHIMURA, E. M. Física das radiações. São Paulo: Oficina de Textos, 2010.

PINTO, A. C. B. C. F.; DIAS, M. T. P .; SANTOS, A. C .; MELO, C. S; FURQUIM,T. A. C. Análise preliminar das doses para avaliação da qualidade da imagem em exames radiográficos na Radiologia Veterinária. Revista Brasileira de física médica . v. 4, n. 1, 2010.

RAMPAZZO, V; FRIOLANI, M.; CAMARGOS, A. Trauma torácico em cães - relato de caso. Revista científica eletrônica de medicina veterinária. v. XI, n. 20 , jan. 2013.

TEODORO, A. A importância da Radiologia na Medicina Veterinária. Revista CRTRSP. $35^{\text {a }}$ ed., p 6-8, set. 2007.

THRALL, D. Diagnóstico de radiologia veterinária. Rio de Janeiro: Elsevier, 2014.

VETTORATO, M. C.; FERNANDES, M. A. R.; VULCANO, L. C.; e FOGAÇA, J. L.. Principais avanços e aplicações da radioterapia na medicina veterinária. Tekhne e Logos, v. 8, n. 1, p. 103-118, 2017. Disponível em: <http://fatecbt.edu.br/seer/index.php/tl/article/view/455> Acesso em: 18 jul. 2019. 